\title{
Uphill solitary waves in granular flows
}

\author{
E. Martínez, C. Pérez-Penichet, O. Sotolongo-Costa, ${ }^{1}$ O. Ramos, K. J. Måløy, ${ }^{2}$ S. Douady, ${ }^{3}$ and E. Altshuler ${ }^{4}$, * \\ 1 "Henri Poincaré" Group of Complex Systems, Physics Faculty, University of Havana, 10400 Havana, Cuba \\ ${ }^{2}$ Physics Department, University of Oslo, Blindern, N-0316 Oslo, Norway \\ ${ }^{3}$ Laboratoire Matire et Systmes Complexes (MSC), \\ Universit Paris 7 - Denis Diderot / CNRS, CC 705675251 Paris Cedex 05 - France \\ 4 "Henri Poincaré" Group of Complex Systems and Superconductivity Laboratory, \\ Physics Faculty-IMRE, University of Havana, 10400 Havana, Cuba
}

(Dated: September 13, 2018)

\begin{abstract}
We have experimentally observed a new phenomenon in the surface flow of a granular material. A heap is constructed by injecting sand between two vertical glass plates separated by a distance much larger than the average grain size, with an open boundary. As the heap reaches the open boundary, "soliton-like" fluctuations appear on the flowing layer, and move "up the hill" (i.e., against the direction of the flow). We explain the phenomenon in the context of stop-and-go traffic models, and show that soliton-like behavior is allowed within a Saint-Venant description for the granular flow.
\end{abstract}

PACS numbers: 45.70.-n,45.70Mg,45.70.Vn,81.05Rm,89.75.-k

The rich dynamics of granular matter -studied for centuries by engineers- has attracted much attention from the Physics community since the early 1990's 1, 2, 3]. Granular flows, for example, have concentrated intense interest, due to their relevance to natural avalanches and industrial processes, and also because they make liquidlike and solid-like behaviors coexist. They have been theoretically described based on the existence of two phases: the rolling (or flowing) one, and the static one. Such idea has been casted into ad hoc phenomenological equations [4, 5, 6], into a Saint-Venant hydrodynamic approach conveniently modified to take into account the particularities of granular matter 7, 8], and eventually by defining an order parameter characterizing the local state of the system [9, 10]. Finally, granular flows have been described by "microscopic" equations based on the newtonian motion of individual grains submitted to gravity, shocks, and trapping events [1, 12].

In particular, researchers have studied both experimentally and theoretically granular flows on inclined planes and tubes, and granular heaps, finding a whole jungle of patterns, spatio-temporal structures, and other nontrivial phenomena, such as fingering 13], avalanches extending both downhill and uphill 14], "logitudinal vortices" 15, 16], "bubbling flows" 17, 18, "revolving rivers" [19] and even "singing" dunes [20].

So it looks very unlikely that one can find further unexplored phenomena in experiments as simple as pouring sand on a heap with an open boundary. However, we report here the existence of soliton-like waves moving uphill in such experiments, i.e., the spontaneous appearance of bump-like instabilities in the flowing layer that propagate uphill, contrary to the flow of sand.

We used sand with a high content of silicon oxide and an average grain size of $100 \mu \mathrm{m}$ from Santa Teresa (Pinar del Río, Cuba) [19]. The sand was poured into a cell consisting in a horizontal base and a vertical wall, sand-

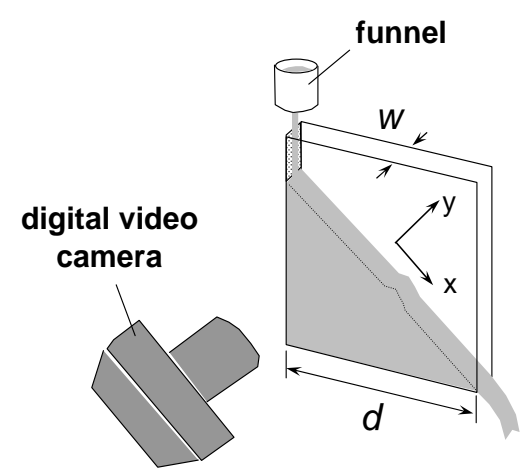

FIG. 1: Simplified diagram of the experimental setup

wiched between two square glass plates with inner surfaces separated by a distance $w$ in the range from $0.3 \mathrm{~cm}$ to $3 \mathrm{~cm}$ (Fig. 1). The lengths of the base and the vertical wall were approximately $d \approx 23 \mathrm{~cm}$. The sand was poured vertically into the cell near the vertical wall using tiny funnels with several hole diameters, in order to obtain different flux values. As the sand was poured into the cell, a heap grew until it reached the open boundary, where the grains were allowed to fall freely (Fig. 1). Digital videos were taken using a High Speed Video Camera Photron FASTCAM Ultima-APX model 120K in the range from $50 \mathrm{fps}$ to $4000 \mathrm{fps}$, with a resolution of $1024 \times 1024$ pixels.

Fig. 2 contain images from experiments with $w=$ $0.7 \mathrm{~cm}$ and $F=0.6 \mathrm{~cm}^{3} / \mathrm{s}$. Fig. 2 (a) is a picture taken from a video recorded at $50 \mathrm{fps}$, and show a closeup of a section of the surface at the central region of the heap, where our basic findings are easily identified: "bumpshaped" instabilities appearing at random places of the surface move "up the hill", as indicated by the white arrows. These bumps could travel either alone or in tandem. The bumps maintain their "identity" within variable times, which can reach more than 10 seconds. After 


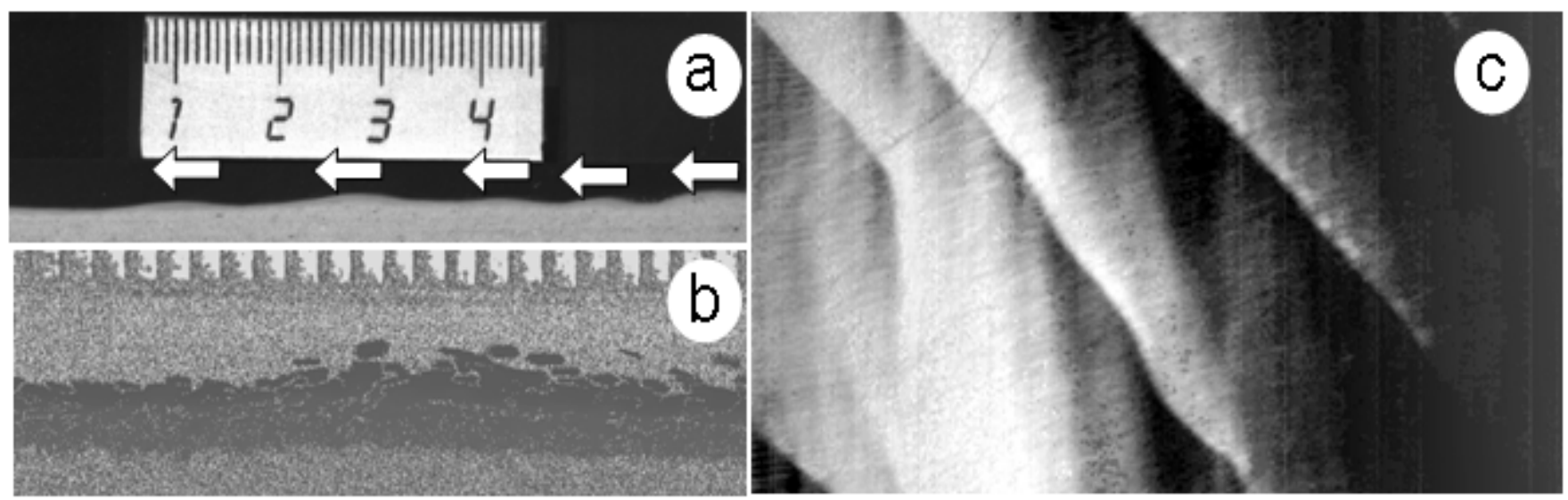

FIG. 2: Basic experimental findings. (a) Uphill bumps moving in tandem, identified by arrows. (b) Difference between two pictures of a single bump, separated by $50 \mathrm{~ms}$. In both pictures, the smallest ticks in the scales correspond to $1 \mathrm{~mm}$. (c) Spatial-temporal diagram of bump dynamics, where $x$ grows to the right and $t$ grows upward, spanning $20 \mathrm{~cm}$ and $2 s$, respectively.

that, they flatten out. We have also observed that, if two of them move at speeds different enough to interfere with each other, they can be still identified after the interaction. However, these are rare events, and we have no video record of them. All in all, our observations hint at a soliton-like behavior in the observed instabilities.

Fig. 2(b) shows the difference between two pictures from a single bump taken from a video recorded at $500 \mathrm{fps}$, which are separated $50 \mathrm{~ms}$ from each other. The darker horizontal band allows to visualize the downhill motion. Careful examination of picture 2(b) allows to separate an approximately $1 \mathrm{~mm}$-depth band of "flying" grains, and an approximately $1 \mathrm{~mm}$ depth layer of "flowing" grains underneath. The height of the perturbation above the unperturbed stream is difficult to measure, but it can be roughly estimated as $1 / 5$ of the flowing layer.

Fig. 2(c) shows a spatial-temporal diagram of the free surface. A horizontal line of the video record was taken just at the (average) free surface, so lower parts appear black, and higher parts appear white. From the picture it can clearly be seen four shock-waves, passing suddenly from black to white, moving upward with a well defined velocity (even if it fluctuates a little on the right). On the left the appearance of a new soliton can be seen, first increasing in height before starting suddenly to propagate.

The average speed of the uphill motion of the bumps as a function of the input flux, $F$ was estimated from the analysis of the motion of several instabilities using pictures analogous to those used to obtain Fig 2(b). Fig. 3 presents the dependence of the average uphill speed versus input flux. The solid line corresponds to $v_{u p} \sim$ $F^{1 / 4}$, which will be discussed below.

Fig. 4 shows a sequence of pictures of a bump separated by intervals of $0.125 \mathrm{~s}$, extracted from a video taken at $4000 \mathrm{fps}$. Careful inspection of the video associated to

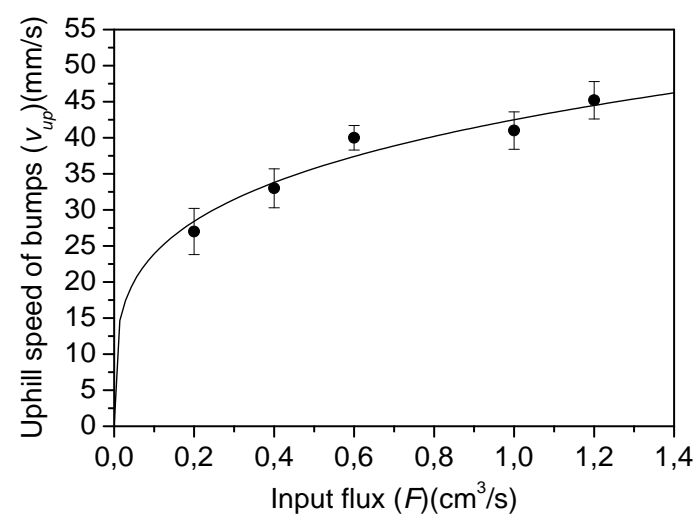

FIG. 3: Uphill speeds of bumps measured as a function of input flux, for $w=0.7 \mathrm{~mm}$

this sequence of images reveals the mechanism of movement of the instability at the single grain level. The grains involved in the flowing layer in picture (a) show two types of behavior: while most of the grains keep flowing from left to right at an average speed $v_{\text {flow }} \sim 10 \mathrm{~cm} / \mathrm{s}$ as the bump passes underneath from right to left, a fraction of the grains at the lower part of the flowing layer show a different behavior. The white circle in Fig. 4(a), represents one of those grains, which is moving from left to right. From 4(b) to 4(d), the grain has incorporated into the static layer (deposition), while the bump is passing from right to left above it. In Fig. 4(e), the grain has reincorporated into the flowing layer (erosion), moving again from left to right.

Exchange of particles between the static and fluid phases has been considered in several models of surface flows since the 1990's [4. We believe that our bumps form when a fluctuation implies an extra deposition of grains from the flowing layer on the static one, which then 


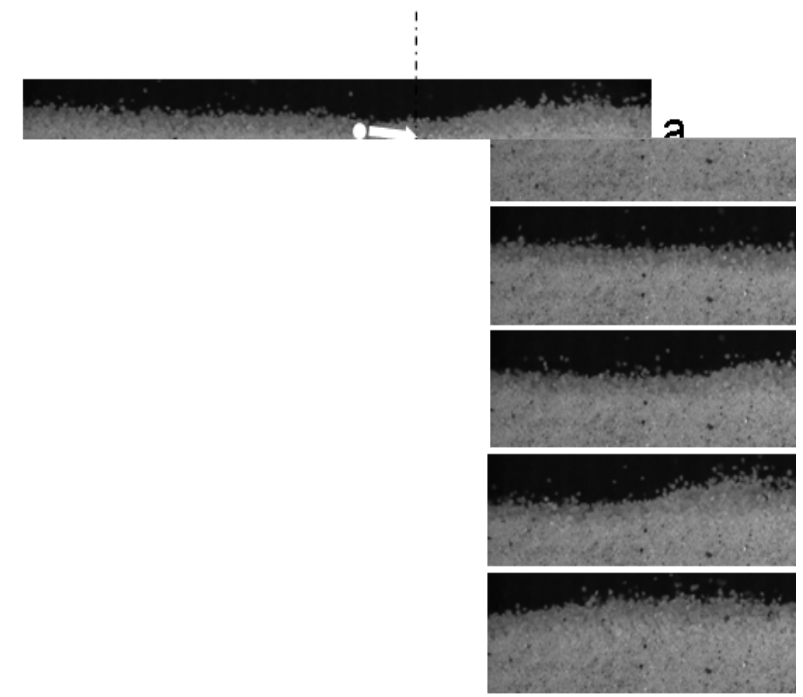

FIG. 4: Sequence of pictures of an bump moving uphill, with the same parameters described for Fig. 2(e). The white circle represents a grain of sand (a) moving downhill as part of the flowing layer, (b)-(d) trapped in the static layer as the bump passes by from left to right, and (e) moving again as part of the flowing layer. The horizontal length of the picture corresponds to $22 \mathrm{~mm}$ in reality

propagates backwards through an "stop-and-go" mechanism observed in traffic dynamics 22. However, in our case the usual traffic models should be modified to take into account the free surface. A way to do it could be a two-lane system consisting in a fast lane where cars flow steadily, and a slow lane where cars stop and go, producing a backward wave (or train of waves) within the slow lane, well described in the literature [22].

All in all, the situation can be pictured as a solitary wave crawling uphill underneath a shallow stream of sand flowing downhill. In fact, the stop-and-go traffic model has been mapped into the "classical" Kortweg-de Vries $(\mathrm{KdV})$ equation after a few approximations, resulting in soliton-like solutions 23. In the context of the $\mathrm{KdV}$ equation, the speed of the soliton can be estimated as $v_{s} \sim \sqrt{g h_{0}}$ where $g$ and $h_{0}$ are the acceleration of gravity and the depth of the unperturbed stream, respectively 24]. If one assumes the well accepted result that the depth of the flowing layer is proportional to $F^{1 / 2}$ [21], we then get $v_{s} \sim F^{1 / 4}$, which follows the experimental result shown in Fig. 3.

Knowing that the KdV equation can be derived from mass and momentum conservation equations applied to an incompressible fluid flowing down a shallow channel 24], we could expect that soliton solutions can be found if we describe our system through hydrodynamics SaintVenant equations modified to take into account the particularities of granular matter [7, 8] which we are writing relative to a reference frame parallel to the average free

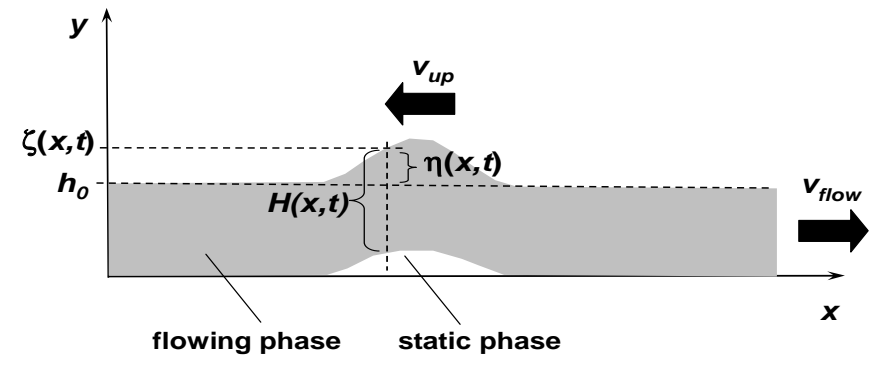

FIG. 5: Diagram showing the neighborhood of a "bump" which includes some parameters of the Saint-Venant model described in the text.

surface of the heap (see Fig. 5) . The first equation corresponds to mass conservation:

$$
\partial_{t} \zeta+\partial_{x}\left(\Gamma H^{2} / 2\right)=0
$$

where $\zeta$ is the height of the free surface, $H$ is the thickness of the flowing layer, and $\Gamma$ is the velocity gradient transversal to the flowing layer, which is assumed constant. Then, the momentum $q=\Gamma H^{2} / 2$ evolves with $H$ due to the erosion/deposition process, like the one illustrated for one grain in Fig. 4. The second equation corresponds to the conservation of momentum, which can be seen as an equation governing the evolution of the thickness of the flowing layer, $H$ :

$$
\partial_{t} H+\partial_{x}\left(\Gamma H^{2} / 2\right)=\frac{g}{\Gamma}(\tan \theta-\mu(H))
$$

where $\mu(H)$ is the friction acting on the layer and $\tan \theta=-\partial_{x} \zeta$ is the slope of the free surface.

Let us assume that that the friction coefficient depends on $H$ in the general form:

$$
\mu=\alpha+\left(\beta+\frac{\gamma}{H}\right)\left(h_{0}^{2}-H^{2}\right) \sqrt{\delta-\varepsilon\left(h_{0}^{2}-H^{2}\right)}
$$

where the values of constants $\alpha, \beta, \gamma, \delta$ and $\epsilon$ can be expressed in terms of experimental parameters $v_{\text {up }}, v_{\text {flow }}$, $h_{0}, g$, and a characteristic length $L_{0}$. Then, equations (1) and (2) can be transformed into the "textbook" KdV equation [24]

$$
L_{0}^{2} v_{f l o w} \eta^{\prime \prime}+6\left(v_{u p}-v_{f l o w}\right) \eta+30 \frac{v_{f l o w}}{h_{0}} \eta^{2}=0
$$

In (4), $\eta=\zeta-h_{0}$ and $\eta^{\prime \prime}=\frac{d^{2} \eta}{d \varphi^{2}}$, where $\varphi=x+v_{u p} t$. One particular solution of that equation, is

$$
\zeta=h_{0}+\frac{h_{0}}{5} \operatorname{sech}^{2}\left(\frac{x+v_{u p} t}{L_{0}}\right)
$$


where we have taken $L_{0}=10 h_{0}, v_{\text {flow }}=\frac{1}{2} \Gamma h_{0}=$ $\sqrt{g h_{0}}$, and $v_{u p}=\frac{1}{3} v_{\text {flow }}$ in order to match our experimental observations (i.e., mainly that the uphill speed is roughly $1 / 3$ of the flow speed, and the height of the perturbation is approximately $1 / 5$ of the thickness of the unperturbed flowing layer). Formula (5) describes a "textbook", bell-shaped soliton moving upward. This solution holds for a friction coefficient given by equation (3) which is a positive, decreasing function of $H$ when reasonable experimental parameters are introduced, in agreement with previous models [8]. Although we do not discard that "non-textbook" solitonic equations might describe the observed bumps without imposing such strong constraints, we stress that our objective here is to show that "textbook" solitons can be derived if the system displays an appropriate $\mu(H)$.

We now present a number of additional experimental facts relative to our uphill soliton waves. First, they have been observed in the whole range of experimental parameters explored, i.e. $0.15 \mathrm{~cm}^{3} / \mathrm{s} \leq F \leq 3 \mathrm{~cm}^{3} / \mathrm{s}$ and $0.3 \mathrm{~cm} \leq w \leq 3 \mathrm{~cm}$. As many phenomena in granular matter, the uphill waves depend, to some extent, on the way the heap is prepared, and show some degree of "memory". When the heap is prepared from scratch, uphill bumps nucleate at random places near the center of the heap, and, within a few seconds, they typically appear near the open boundary. If the experiment is stopped and re-started in such conditions, solitons reappear immediately. That happens even if a layer of sand less than $1 \mathrm{~mm}$-thick is removed from the surface. If a thicker layer is removed, one has to wait a few seconds to observe them. This is probably due to the formation of a "compactified" layer during the flow that is necessary for the formation of these "jammed" bumps. Finally, all the results we have presented here have been observed just for one type of sand that produces sandpiles through "revolving rivers" when dropped on a flat horizontal surface [19]. This fact supports the idea that a quite specific $\mu(H)$ dependence is needed to observe the solitary waves we report in this paper.

In summary, we have observed soliton-like instabilities in a flow of sand established on a heap with open boundaries, moving against the direction of the flow. The phenomenon can be understood in the light of stop-and-go traffic arguments, even for this free surface flow, and can be described by Saint-Venant equations adapted to granular flows. The "microscopic" mechanisms that make a certain sand more suitable to show uphill bumps remains a mystery, but it seems clear that the dependence of the friction coefficient of our particular sand on the flowing depth is appropriate for the appearance of solitons.

We thank $\varnothing$. Johnsen and C. Noda for help in experi- ments and image processing, and E. Clèment, A. Daerr, S. Franz, H. Herrmann, J. Marín, D. Martínez, R. Mulet, D. Stariolo and J.E. Wesfreid for useful discussions and comments. G. Quintero and J. Fernández collaborated in numerical calculations. We appreciate financial support from the "Abdus Salam" ICTP during the last stage of this project.

* ea@infomed.sld.cu

[1] H.Jaeger, S. R. Nagel and R. P. Behringer, Rev. Mod. Phys 68, 1259 (1996)

[2] P. G. DeGennes, Rev. Mod. Phys 71, S374 (1999)

[3] L. Kadanoff Rev. Mod. Phys 71, 435 (1999)

[4] J. P. Bouchaud, M. E. Cates, J. Ravi Prakash and S. F. Edwards, J. Phys. I 4, 1383 (1994)

[5] P. G. de Gennes, C. R. Acad. Sci., Ser. IIb: Mech., Phys., Astron. 321, 501 (1995)

[6] A. Aradian, E. Raphael and P. G. de Gennes Phys. Rev. E. 60, 2009 (1999)

[7] S. Douady, B. Andreotti and A. Daerr Eur. Phys. J. B 11, 131 (1999)

[8] B. Andreotti, A. Daerr, and S. Douady Phys. Fluids 14, 415 (2002).

[9] I. S. Aranson and L. S. Tsimring, Phys. Rev. E. 64, 020301 (2001)

[10] I. S. Aranson and L. S. Tsimring, Phys. Rev. E. 65, 061303 (2002)

[11] L. Quartier, B. Andreotti, S. Douady and A. Daerr Phys. Rev. E. 62, 8299 (2000)

[12] B. Andreotti and S. Douady Phys. Rev. E. 63, 031305 (2001)

[13] O. Pouliquen, J. Delour and S. B. Savage Nature 386, 816 (1997)

[14] A. Daerr and S. Douady Nature 399, 241 (1999)

[15] Y. Forterre and O. Pouliquen Phys. Rev. Lett. 86, 5886 (2001)

[16] Y. Forterre and O. Pouliquen J. Fluid Mech. 467, 361 (2002)

[17] E. G. Flekkøy, S. McNamara, K. J. Måløy and D. Gendron Phys. Rev. Lett. 87, 134302 (2001)

[18] D. Gendron, H. Troadic. K. J. Mål( $\varnothing)$ y and E. Flekkøy Phys. Rev. E 64, 021509 (2001).

[19] E. Altshuler, O. Ramos, E. Martínez, A. J. BatistaLeyva, A. Rivera, and K. E. Bassler, Phys. Rev. Lett. 91, 014501 (2003).

[20] S. Douady, A. Manning, P. Hersen, H. Elbelrhiti, S. Protiere, A. Daerr, B. Kabbachi, arxiv.org/abs/nlin/0412047v1

[21] G. D. R. Midi, Eur. Phys. J. E 14, 341 (2004)

[22] D. Helbing, Rev. Mod. Phys. 73, 1067 (2001).

[23] P. Berg, A. Woods, Phys. Rev. E. 64, 035602 (2001).

[24] M. Toda, Nonlinear Waves and Solitons, Kluwer Academic Publishers, Dordrecht, 1989 